\title{
SIMULACIÓN 3D DEL FLUJO EN UN COMPRESOR CENTRÍFUGO POR MEDIO DE HERRAMIENTAS CFD BAJO CARGA PARCIAL
}

\section{D FLOW SIMULATION IN A PARTIALLY LOADED CENTRIFUGAL COMPRESSOR USING CFD TOOLS}

\author{
Jairo Andrés Acosta Rojas ${ }^{1} \quad$ Carlos Alberto Duque Daza ${ }^{2}$ \\ Miguel Mantilla González ${ }^{3}$ Carlos Humberto Galeano Urueña ${ }^{4}$ \\ Recibido el 14 de junio de 2007, aceptado el 19 de diciembre de 2007 \\ Received: June 14, 2007 Accepted: December 19, 2007
}

\begin{abstract}
RESUMEN
El presente artículo presenta el procedimiento llevado a cabo para simular el flujo de aire en un compresor centrífugo HOLSET HT3B por medio de FLUENT $^{\circledR}$ y GAMBIT ${ }^{\circledR}$, paquetes de CFD (Computer Fluid Dynamics) basados en la técnica de volúmenes finitos. Inicialmente se presentan algunas consideraciones importantes que deben tenerse en cuenta al usar las herramientas que este método ofrece para la simulación de flujos. Posteriormente se describirá el proceso seguido para obtener los resultados preliminares de la simulación y se discutirán brevemente las características más relevantes de los mismos. Es importante mencionar que este artículo hace referencia a la simulación inicial del flujo en el compresor mencionado, y que uno de sus principales aportes es el de hacer recomendaciones para la realización del ajuste de los parámetros de operación escogidos.
\end{abstract}

Palabras clave: CFD, compresores centrífugos, simulación numérica, volúmenes finitos.

\section{ABSTRACT}

This article presents the procedure followed to simulate the air flow in a HOLSET HT3B centrifugal compressor using FLUENT ${ }^{\circledR}$ and GAMBIT ${ }^{\circledR}$, both CFD (Computer Fluid Dynamics) packages, which are based on the finite volume technique. Initially some important considerations will be presented, which must be considered when using the CFD tools for the flow simulation; later, the simulation process will be described to obtain the first results, and their most relevant characteristics will be discussed briefly. It is important to mention that this article makes reference to the initial simulation of the flow in the mentioned compressor, and that some recommendations for the operational parameters adjustments are presented as a plus.

Keywords: CFD, centrifugal compressors, numerical simulation, finite volume.

\section{INTRODUCCIÓN}

La presente simulación hace parte de las actividades de investigación y apropiación en simulación de campos de flujo, emprendidas en el marco del proyecto de investigación "Formulación y Evaluación de un Sistema Alterno de Generación Local de Energía a partir de Microturbinas", el cual se desarrolla actualmente con el apoyo del departamento de Ingeniería Mecánica y Mecatrónica de la Universidad Nacional de Colombia (DIMM). De forma previa se han desarrollado, dentro del DIMM, modelos CFD de flujos en turbomaquinaria para diferentes aplicaciones, los cuales debido a distintas limitaciones se han restringido a la simulación en dos dimensiones de tales flujos. En este artículo se describe el primer acercamiento hecho en el DIMM a simulaciones tridimensionales de flujos en turbomaquinaria, con metodologías de aplicación académica mediante el uso de herramientas CFD comerciales.

De esta forma, con esta simulación se busca comenzar a construir conocimiento en torno al manejo de modelos tridimensionales en paquetes de CFD comerciales como FLUENT $^{\circledR}$ y GAMBIT $^{\circledR}$, y particularmente en torno al manejo de modelos de compresores centrífugos de la escala de los turbocargadores empleados en vehículos automotores.

\footnotetext{
1 Universidad Nacional de Colombia. Facultad de Ingeniería. Kr. 30 \# 45-03, Edif. 453 Of. 401. Bogotá, Colombia. E-mail: jaacostaro@ unal.edu.co 2 Universidad Nacional de Colombia. Facultad de Ingeniería. Kr. 30 \# 45-03, 453-401. Bogotá, Colombia. E-mail: caduqued@unal.edu.co. Autor corresponsal.

3 Universidad Nacional de Colombia. Facultad de Ingeniería. Kr. 30 \# 45-03, Edif. 453 Of. 401. Bogotá, Colombia. E-mail: jmmantillag@unal.edu.co

4 Universidad Nacional de Colombia. Facultad de Ingeniería. Kr. 30 \# 45-03, Edif. 453 Of. 401. Bogotá, Colombia. E-mail: chgaleanou@ unal.edu.co
} 
El objeto de este artículo es presentar la metodología que ha sido utilizada para la simulación tridimensional del flujo de aire a través del compresor centrífugo de un turbocargador HOLSET HT3B, indicando los parámetros y las consideraciones que mejores resultados presentaron a lo largo de todo el proceso de simulación.

La descripción de la etapa de modelado geométrico del compresor objeto del presente estudio se trata en [1], de modo que en el presente artículo se omiten los detalles de esta etapa y se presentan pormenores tanto del preprocesamiento del modelo como de su solución y del postprocesamiento de los resultados.

\section{PREPROCESAMIENTO DEL MODELO}

Antes de comenzar a describir el preprocesamiento es necesario presentar el modelo geométrico creado en SolidEdge e importado desde GAMBIT ${ }^{\circledR}$ (programa preprocesador de FLUENT $^{\circledR}$ ), como archivo con formato ACIS y extensión .sat. Dicho modelo se ilustra en la figura 1 .
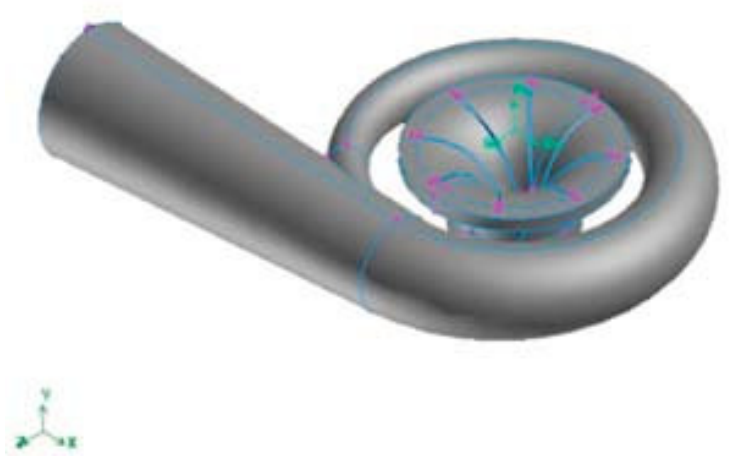

Figura 1. Modelo geométrico importado en Gambit.

El preprocesamiento en GAMBIT ${ }^{\circledR}$ consiste básicamente en la generación de la geometría real, la simplificación del modelo geométrico (Clean up), su posterior mallado y la definición de zonas del mismo. Después de realizar estos pasos debe exportarse el modelo mallado a FLUENT $®$, donde se establecen los parámetros necesarios para iniciar la etapa de solución. A continuación se muestra el desarrollo de cada uno de estos pasos del preprocesamiento.

\section{Geometría Real}

Al observar la figura 1 se evidencia, por el espacio vacío presente entre voluta y rodete, que la sección del difusor que conecta la región de flujo del rodete con la de la voluta no se construye en el modelo geométrico inicial. Este hecho se explica considerando que al construir de esta forma el compresor, se simplifica su posterior división en subregiones de flujo, necesarias para facilitar el mallado del modelo en la etapa de preprocesamiento [2].

GAMBIT $^{\circledR}$ maneja un esquema de modelado que parte de elementos geométricos sencillos (puntos o vértices) para crear elementos geométricos de malla, después de lo cual se generan elementos geométricos de mayor complejidad (líneas, superficies y volúmenes). Con esto presente, la generación del difusor consiste en: la ubicación de los puntos importantes para su construcción, la conexión de estos puntos por medio de rectas, la creación, a partir de las líneas, de superficies que sirven de frontera para el difusor, para finalmente crear el difusor como volumen. De esta manera se genera una sección de mayor extensión que la requerida, debido a que la intersección que se hubiese dado entre la voluta y el difusor, al construir la sección transversal al final de este último, induce errores en el preprocesador GAMBIT ${ }^{\circledR}$. Es de notar que en vez de generar en un solo paso la sección transversal del difusor, se construye un volumen base que posteriormente es editado por medio de operaciones booleanas. El modelo final obtenido en GAMBIT $^{\circledR}$ se presenta en la figura 2.

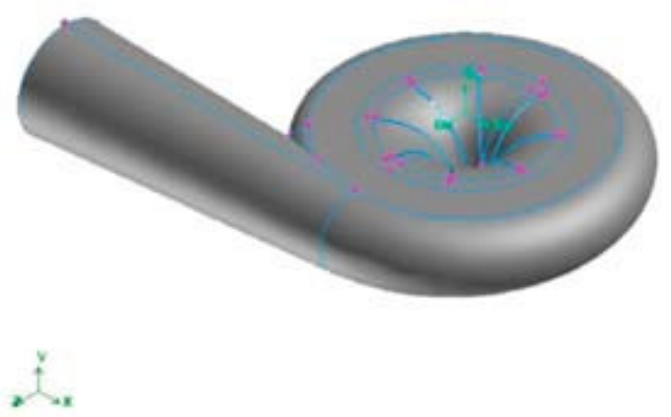

Figura 2. Modelo final obtenido en Gambit.

Una vez que se construye el modelo completo del compresor, éste se divide en tres zonas: la zona correspondiente al rodete se separa en dos volúmenes, debido a que así se facilita el mallado. A su vez estas dos regiones se separaron de la zona estática del compresor.

\section{Clean Up}

El uso de la opción ofrecida por GAMBIT®, denominada Geometría Virtual, es el segundo paso en el modelado de zonas de flujo de geometría compleja importadas desde un programa de CAD, y se lleva a cabo con el objetivo de eliminar los errores inducidos en el modelo geométrico durante la importación desde el software de origen [3]. En el desarrollo del presente trabajo se han encontrado dos 
tipos de defectos geométricos: líneas o bordes demasiado cortos y superficies demasiado pequeñas. Inicialmente se eliminan las líneas que son demasiado cortas usando la opción Vertex Connect, buscando las líneas más cortas del modelo y determinando si son o no aptas para el mallado del modelo. El segundo defecto encontrado en el modelo geométrico real, relacionado con el tamaño, la simetría y la complejidad de las superficies, se ha abordado usando el comando Merge Faces, con el cual pueden combinarse las superficies antes de ser malladas.

\section{Mallado del modelo}

A partir del trabajo realizado hasta este punto, se llega a determinar que la mejor estrategia de mallado consiste en partir del volumen considerado como el de mayor complejidad de mallado, prosiguiendo en un orden de dificultad descendente. Teniendo en cuenta esta consideración, el primer volumen en ser mallado fue el correspondiente a la sección posterior del rodete, seguida por la sección de entrada al mismo y finalmente por el conjunto difusor-voluta.

Otra consideración importante en la construcción de cada una de las mallas tiene que ver con el control de la "densidad de elementos". Efectivamente, hay regiones del flujo en las que los altos gradientes de presión, velocidad o temperatura llevan a que sea necesario implementar un gran número de elementos pequeños que permitan reproducir adecuadamente las características del flujo en cuestión, sin que surjan problemas de difusión numérica. Para hacer esto, de forma análoga a la utilizada en GAMBIT $^{\circledR}$ para generar el modelo geométrico, es conveniente comenzar a mallar las líneas del modelo que se encuentren en las zonas de flujo que, según se considera, son más críticas para el posterior desarrollo de la simulación (ver figura 3).

Es conveniente anotar que no es necesario mallar todas las líneas del modelo, pues este paso sólo se requiere para controlar o modificar el tamaño de los elementos de la malla en zonas muy específicas, de modo que las regiones que en este paso se han omitido, se mallan según las especificaciones indicadas al momento de discretizar ${ }^{5}$ superficies o volúmenes.

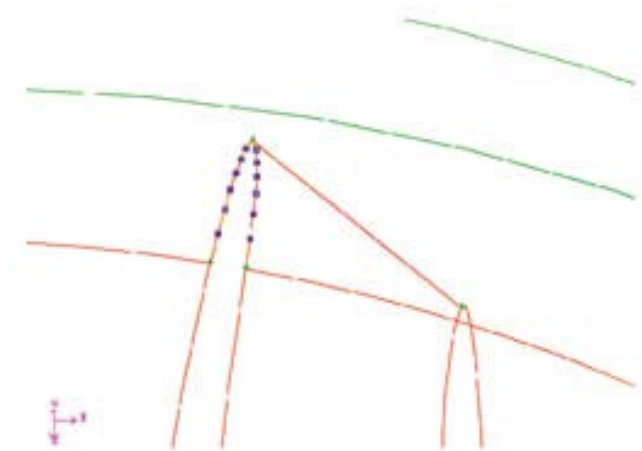

Figura 3. Mallado de líneas en zonas especiales del modelo geométrico.

A partir de las líneas que se dividen para controlar la densidad de los elementos de la malla en regiones críticas del modelo, se procede a discretizar las superficies del mismo. Para este caso la generación de la malla 3D final del rodete requiere que todas las superficies sean divididas previamente, de modo que se pueda ejercer un control efectivo de la malla sobre todo el volumen. Las superficies de los álabes se discretizan con elementos cuadrilaterales (lo cual permite que se obtenga una mayor fidelidad de los efectos de capa límite sobre los álabes [3]) y las demás superficies con elementos triangulares, usando en ambos casos el método Pave $e^{6}$.

El éxito de la discretización de cada uno de los volúmenes que componen el compresor radica básicamente en las características de las mallas de las superficies del volumen y del esquema de mallado utilizado. En GAMBIT ${ }^{\circledR}$, el esquema TGrid es el más adecuado para trabajar con regiones de flujo de geometría compleja, debido a que crea mallas no estructuradas con elementos tetrahedrales y piramidales. Finalmente, se obtiene una malla con 1058042 elementos, la cual se muestra en la figura 4.

\section{Definición de zonas en el modelo}

Antes de exportar el archivo de la malla a FLUENT ${ }^{\circledR}$ para realizar la simulación, se hace necesario establecer la naturaleza de cada una de las fronteras del modelo geométrico y del volumen en sí. En este proyecto se han incluido las zonas presentadas en la tabla 1 .

\footnotetext{
5 La palabra discretizar se emplea a lo largo del artículo como un sinónimo de la palabra mallar.
}

\footnotetext{
6 Pave es un esquema de mallado proporcionado por GAMBIT® ${ }^{\circledR}$ para la creación de mallas no estructuradas sobre superficies regulares o irregulares.
} 


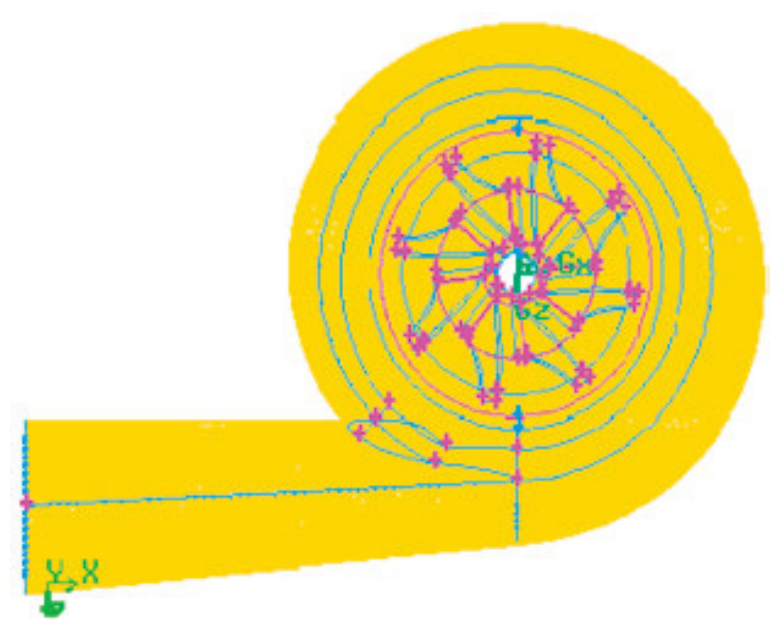

Figura 4. Malla 3D del modelo geométrico del compresor.

Debido a que en la simulación objeto de este proyecto se tienen dos zonas de flujo con diferentes marcos de referencia (uno estático y uno en rotación), también es necesario definir la sustancia ubicada en el espacio demarcado por cada una de tales zonas, como se especifica en la tabla 2.

Tabla 1. Determinación de las condiciones de frontera en GAMBIT.

\begin{tabular}{|l|l|}
\hline NOMBRE DE ZONA & \multicolumn{1}{|c|}{ TIPO } \\
\hline Entrada & Pressure Inlet \\
\hline Salida & Pressure Outlet \\
\hline Interface $^{\mathrm{B}}$ & Wall \\
\hline Álabes & Wall \\
\hline Hub & Wall \\
\hline Shroud & Wall \\
\hline Wall & Wall \\
\hline
\end{tabular}

A Los nombres dados a las zonas son elegidos por el usuario, sin que esto implique condición alguna en la frontera nombrada.

B Zona ubicada entre las regiones estática y dinámica del modelo.

C Superficies restantes del modelo.

Tabla 2. Definición de los volúmenes del modelo.

\begin{tabular}{|c|c|}
\hline ZONA & TIPO \\
\hline Carcasa & Fluid \\
\hline Rodete & Fluid \\
\hline
\end{tabular}

\section{Preparación de la simulación}

Pruebas preliminares permitieron definir los parámetros de simulación que ofrecen los mejores resultados, éstos se resumen en la tabla 3 .
Tabla 3. Definición de parámetros en FLUENT.

\begin{tabular}{|c|c|}
\hline PARÁMETRO & DEFINICIÓN \\
\hline Unidades & SI \\
\hline Solver & Segregated \\
\hline Linealización & Explicit \\
\hline Régimen & Estacionario \\
\hline Viscosidad & k- $\varepsilon$ Estándar [4] y [5] \\
\hline Materiales & $\begin{array}{l}\text { - Inicialización de la simulación: } \\
\text { Aire de densidad constante. } \\
\text { - Finalización de la simulación: } \\
\text { Aire, gas ideal. }\end{array}$ \\
\hline
\end{tabular}

Antes de continuar presentando las condiciones de operación y de frontera del modelo, es conveniente aclarar las razones por las que se utilizaron dos tipos diferentes de fluido de trabajo; éstas se relacionan con la metodología empleada. En un paso inicial, la densidad del aire en el compresor se considera constante, dejando de lado los cambios de presión debidos a las variaciones de temperatura y densidad del fluido, obteniéndose estimaciones iniciales de estas variables a partir de los cambios de velocidad. La ventaja de implementar esta suposición inicial radica en que la solución del modelo se limita al trabajo con dos ecuaciones de conservación: la de momentum y la de continuidad, es decir, se deja de lado momentáneamente la ecuación de energía. Con estas suposiciones se comienza la solución del modelo buscando principalmente un punto de partida adecuado, para después incluir, a partir de éste, la naturaleza compresible del aire, indicando a FLUENT ${ }^{\circledR}$ que la densidad del mismo está definida por la ecuación de los gases ideales. Una vez cambiado este parámetro, el programa integra automáticamente la ecuación de energía a las otras dos ecuaciones de conservación resueltas previamente $\mathrm{y}$, tras ser ejecutada por segunda vez la simulación, se obtiene una solución convergente.

En cuanto a las condiciones de operación, se refiere la presión atmosférica local definida en $75 \mathrm{kPa}$, valor de la presión en la ciudad de Bogotá (Colombia), lugar donde se realizará una posterior validación de los resultados obtenidos.

Las definiciones más importantes a tener en cuenta en el momento de especificar las condiciones de frontera se relacionan con el movimiento relativo entre las zonas de flujo correspondientes al rodete y a la carcasa. En la presente simulación solamente se utiliza el esquema MRF (Moving Reference Frame), que es un método esencialmente estacionario, donde los resultados que se obtienen dependen en gran medida de la posición de las partes móviles con respecto a las estáticas [4]. Sin embargo, [4-5] afirman que los resultados obtenidos por 
el esquema $M R F$ ofrecen una aproximación satisfactoria para sus respectivos casos de estudio. Para más información respecto a las condiciones de frontera a especificar para implementar el esquema $M R F$ en la simulación, pueden consultarse $[2,6]$.

La velocidad del rodete y del aire en la zona de flujo correspondiente al mismo se especifica en $25000 \mathrm{rpm}$, un valor muy inferior al de la velocidad de trabajo del compresor de un turbocargador HOLSET HT3B. Esta decisión se ha tomado dado que [6] reporta obtención de resultados convergentes y adecuados para las condiciones de trabajo definidas con bajas velocidades de rotación.

\section{SOLUCIÓN DEL MODELO}

La inicialización de la solución se lleva a cabo tomando los valores establecidos por FLUENT ${ }^{\circledR}$ por defecto. Como se ha dicho anteriormente, la metodología de solución se divide en dos partes: una llevada a cabo con la suposición de aire con densidad constante y otra con la suposición de aire como gas ideal.

Dado que la velocidad de convergencia en modelos CFD es fuertemente dependiente de los coeficientes de relajación utilizados para atenuar los efectos no lineales de algunas de las variables del fluido, ha sido necesaria la implementación de una metodología para la selección de dichos valores. En esta metodología se parte de una selección inicial de coeficientes para las variables de la simulación, con los que se llevan a cabo un número determinado de iteraciones empleando el solver Segregated, y llevando a cabo una evaluación de la tasa de convergencia; al cabo de estas, se decide sobre el cambio en los valores establecidos para los coeficientes de relajación, buscando siempre la reducción progresiva de estos valores y su efecto sobre los resultados del modelo. Esta metodología permite alcanzar la convergencia del modelo empleando los coeficientes de relajación presentados en las tablas 4 y 5 . En la tabla 5 se observa que en los resultados obtenidos entre las iteraciones 7.801 a 12.800 no se especifican algunos parámetros de relajación. Esto se debe a que en este intervalo se emplea el solver tipo Coupled con esquema de linealización implícita, cuyas especificaciones difieren de las del solver empleado hasta este punto. Sin embargo, tras hacer este cambio, se observa que el solver Segregated ofrece mejores condiciones durante el proceso de solución (especialmente en la velocidad de convergencia), por lo cual se retomó a partir de la iteración 12801.
La tabla 5 muestra también que a partir de la iteración 12801 se incluye un coeficiente de relajación adicional correspondiente a la ecuación de energía, dado que a partir de esta iteración se cambió el modelo de comportamiento del aire por un esquema compresible. Este cambio tuvo una repercusión acentuada en la convergencia de la solución. En la figura 5 se muestra el monitor de convergencia para las últimas iteraciones del proceso de solución.

Tabla 4. Coeficientes de relajación tomados en el desarrollo de la solución.

\begin{tabular}{|c|c|c|c|c|}
\hline \multirow[b]{2}{*}{ VARIABLES } & \multicolumn{4}{|c|}{ ITERACIONES } \\
\hline & 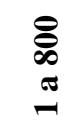 & 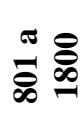 & 串 & 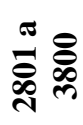 \\
\hline Pressure & 0,2 & 0,2 & 0,2 & 0,2 \\
\hline Density & 1 & 1 & 0,8 & 0,8 \\
\hline Body Forces & 1 & 1 & 1 & 1 \\
\hline Momentum & 0,5 & 0,5 & 0,5 & 0,5 \\
\hline TKE & 0,8 & 0,8 & 0,8 & 0,6 \\
\hline TDR & 0,5 & 0,5 & 0,5 & 0,4 \\
\hline TV & 1 & 1 & 0,8 & 0,6 \\
\hline Energy & - & - & - & - \\
\hline
\end{tabular}

TKE: Turbulent Kinetic Energy; TDR: Turbulence Dissipation Rate; TV: Turbulent Viscosity.

Tabla 5. Coeficientes de relajación tomados en el desarrollo de la solución

\begin{tabular}{|c|c|c|c|c|}
\hline \multirow[b]{2}{*}{ VARIABLES } & \multicolumn{4}{|c|}{ ITERACIONES } \\
\hline & 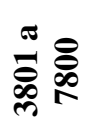 & ๘ & 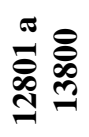 & 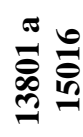 \\
\hline Pressure & 0,2 & - & 0,2 & 0,2 \\
\hline Density & 0,8 & - & 0,8 & 0,7 \\
\hline Body Forces & 1 & - & 1 & 1 \\
\hline Momentum & 0,5 & - & 0,5 & 0,5 \\
\hline TKE & 0,6 & 0,6 & 0,6 & 0,5 \\
\hline TDR & 0,4 & 0,4 & 0,4 & 0,4 \\
\hline TV & 0,6 & 0,6 & 0,6 & 0,5 \\
\hline Energy & - & - & 1 & 1 \\
\hline
\end{tabular}

TKE: Turbulent Kinetic Energy; TDR: Turbulence Dissipation Rate; TV: Turbulent Viscosity. 


\section{POSTPROCESAMIENTO DE RESULTADOS}

\section{Perfiles de presión estática en el rodete}

Según se presenta en las figuras 6 y 7, el lado de presión positiva del rodete se ve sometido a un rango de presiones estáticas manométricas definido entre $0 \mathrm{kPa}$ y $7,12 \mathrm{kPa}$, mientras que el lado de succión se ve sometido a presiones manométricas entre -12,0 kPa y 4,0 kPa, estando la mayor parte de este lado sometido a presiones manométricas negativas. Este grado de compresión está muy por debajo de los niveles requeridos de compresión en turbocargadores [7], en los que generalmente se pueden alcanzar relaciones de compresión mayores a 2 , en tanto que la obtenida en este caso es apenas de 1,1 aproximadamente. Con este resultado, debe recordarse que los parámetros de la simulación definen una velocidad de giro de 25.000 rpm, mientras que la velocidad de operación de un turbocargador de este tipo oscila alrededor de $90.000 \mathrm{rpm}$.

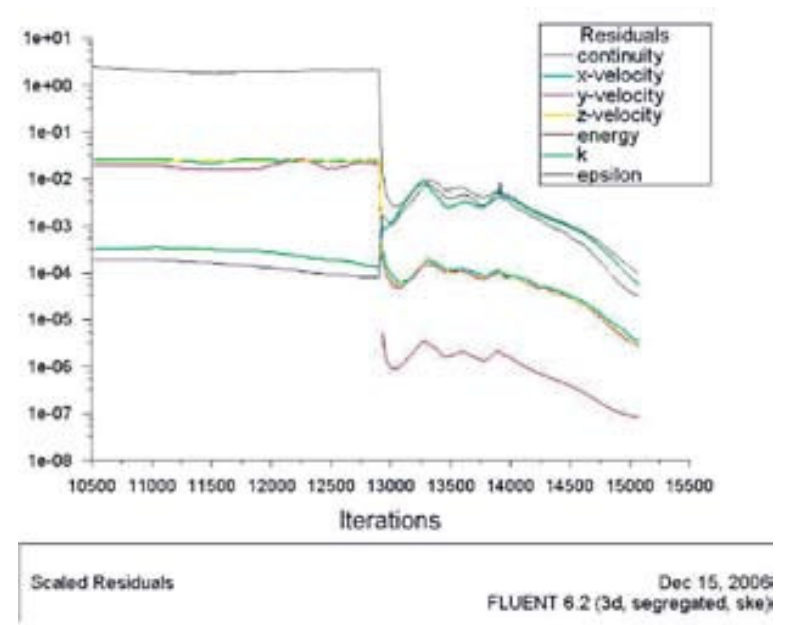

Figura 5. Monitor de convergencia para las iteraciones 10500 a 15106.

Puede también observarse que ninguna de las distribuciones de presión en los casos anteriormente mencionados es completamente simétrica. Este hecho puede ser atribuido a la interacción existente entre las regiones correspondientes al flujo en la voluta y en el rodete, debido a que el cambio paulatino del área de la sección transversal que se tiene en la voluta lleva a que la presión que encuentra el flujo a la salida del rodete varíe en dirección circunferencial [4].

En cuanto al método $M R F$, [6] afirma que es recomendable solamente para casos en los cuales la interacción dada entre las zonas del fluido perturbadas, por elementos dinámicos como el rodete y las zonas estáticas del fluido, es débil. Sin embargo, esto no se cumple en el caso de un compresor centrífugo. A partir de esta consideración puede decirse que se espera que la asimetría de la distribución de presión estática sea subestimada en esta simulación, lo cual puede verificarse ejecutando la simulación del mismo modelo de compresor por medio de un método más apropiado y exacto como lo es el de Sliding Mesh; esta consideración se plantea como una etapa posterior de refinamiento del modelo.

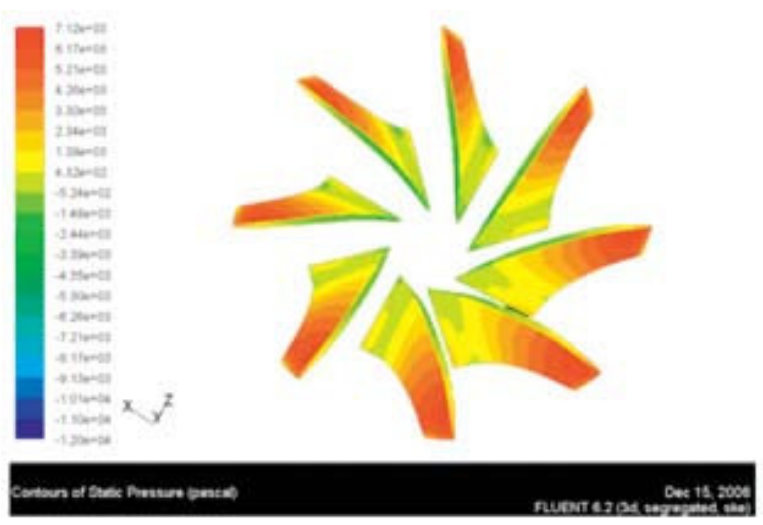

Figura 6. Presión estática en el lado de presión del rodete.

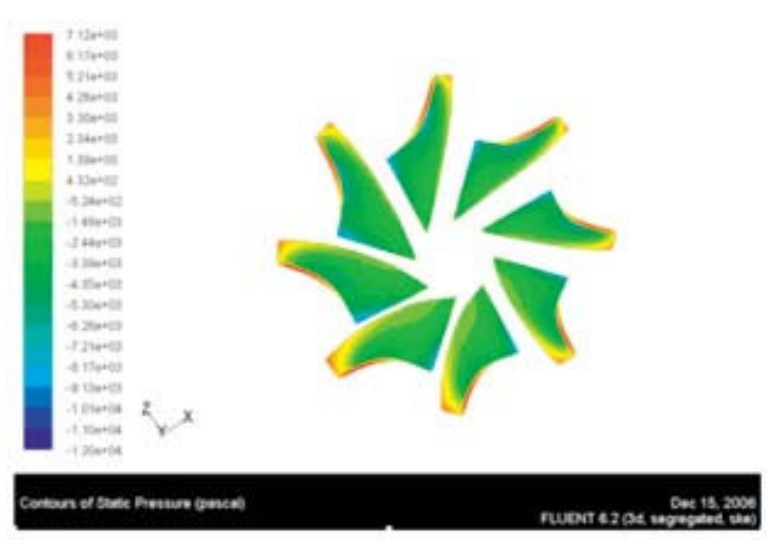

Figura 7. Presión estática en el lado de succión del rodete.

\section{Vectores de velocidad absoluta en el rodete}

Como última parte del postprocesamiento de los resultados, es necesario verificar que los patrones de flujo presentados por los resultados reportados por FLUENT ${ }^{\circledR}$ no presenten reflujo, es decir, zonas en las que el flujo se devuelva hacia la entrada. Para esto puede usarse la gráfica de los vectores de velocidad absoluta, que se muestra en la figura 8 . Se observa que, sobre el plano que corta el difusor a la mitad de su altura, la velocidad absoluta del fluido presenta para todos los canales del rodete una componente de velocidad radial cuyo sentido va desde el centro del rodete hacia fuera. Esta condición demuestra que no se presenta reflujo desde el difusor hacia el rodete. 


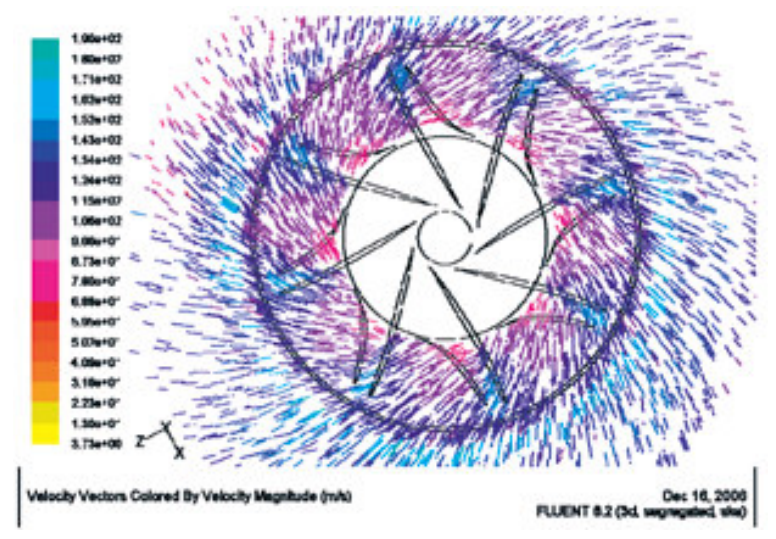

Figura 8. Velocidad absoluta del flujo en la interfaz rodete-voluta.

\section{CONCLUSIONES Y RECOMENDACIONES}

Cada una de las etapas del desarrollo del presente trabajo permite sugerir una serie de recomendaciones que deberían ser tenidas en cuenta para la simulación de compresores centrífugos en general, y sobre todo, para el trabajo que se realice a partir del presente proyecto.

En cuanto al preprocesamiento de la simulación, y particularmente al mallado del modelo geométrico, se puede decir que es una de las tareas que más importancia tiene en toda la preparación de la simulación y que más esfuerzo requiere, sobre todo cuando se trata de una malla tridimensional. Con el fin de simplificar el proceso de mallado para trabajos posteriores, se ve necesario mencionar que la mejor estrategia de mallado consiste en partir del volumen que se estime de mayor complejidad de mallado, prosiguiendo en un orden de dificultad descendente. Asimismo, esta recomendación resulta conveniente en los casos donde se requiera ejercer control sobre la densidad de elementos de la malla.

Por otro lado, haciendo referencia al tema de la simulación realizada, es necesario reconocer que el presente proyecto se constituye como un primer paso en la caracterización de flujo 3D en turbomaquinaria dentro del DIMM. Puesto que este trabajo es uno de los primeros en utilizar las herramientas disponibles en software CFD para la simulación de flujos tridimensionales complejos, dentro de la Universidad Nacional de Colombia, en su realización, se logran reconocer tanto las fortalezas y potenciales, como las restricciones y limitantes de este tipo de software. Esto lleva a que el desarrollo tenga un carácter investigativo, donde las dificultades encontradas en una operación se emplean como elemento de retroalimentación para modificar los parámetros utilizados en tareas previas. Uno de los aportes que hace el presente documento es plasmar la experiencia obtenida en el manejo de este software, de modo que se facilite el trabajo a realizar en el futuro usando CFD como herramienta para simulación de flujos tridimensionales, evitando inconvenientes que surgen básicamente por la falta de experiencia. A partir de este aporte, se espera continuar trabajando en la simulación del mismo turbocargador incrementando la velocidad de trabajo del compresor dentro del modelo computacional, la cual hasta este momento alcanza aproximadamente el $28 \%$ de la velocidad de trabajo en condiciones reales. Vale la pena señalar que, una vez terminada la presente simulación, utilizando los parámetros de solución establecidos para la última serie de iteraciones y partiendo de los resultados de la misma se inicia otra simulación en la que la velocidad de trabajo del compresor se lleva hasta $50000 \mathrm{rpm}$, lo cual representa aproximadamente un $56 \%$ de la velocidad real de operación del turbocargador. Obteniéndose una solución convergente que puede servir de base para el análisis del comportamiento del compresor y la continuación de la caracterización del mismo con FLUENT ${ }^{\circledR}$. Se trabaja adicionalmente buscando incrementar la velocidad de trabajo en la simulación hasta 75000 rpm y 100000 rpm, sin embargo no se obtuvo resultado alguno en ninguno de los casos. Para abordar estas velocidades de rotación debe estudiarse la conveniencia de utilizar el método numérico Coupled y la formulación de velocidad relativa entre otros parámetros más apropiados para manejar altas velocidades de flujo.

Otra tarea a realizar para la caracterización de las fortalezas de FLUENT $^{\circledR}$ (o cualquier otra herramienta CFD) es llevar a cabo la misma simulación utilizando los esquemas de Mixing Plane y de Sliding Meshes para la simulación de zonas en movimiento. Para esto debe iniciarse la simulación a partir de la construcción de la malla, debido a que ésta debe reunir ciertas características especiales para permitir el uso de cada uno de dichos esquemas. A partir de los resultados reportados por grupos de trabajo como [4], puede decirse que el esquema de Mixing Plane puede ser omitido dado que se ha comprobado que no ofrece resultados exactos, mas el esquema de Sliding Meshes debe ser examinado, precisamente, por la calidad de los resultados proporcionados.

Finalmente, es necesario mencionar que CFD es una metodología que aún se encuentra en desarrollo, y que por tanto todavía no es una herramienta en la que se pueda basar un diseño. Entendiendo que los métodos de solución que emplea CFD son aproximaciones numéricas resultantes de una u otra simplificación en los términos de las ecuaciones de Navier-Stokes, y que dependiendo del método numérico escogido se alcanzan ciertos resultados determinados, la validación de los resultados obtenidos por medio del software CFD es absolutamente necesaria. 
El grado de proximidad de dichos resultados a la solución de las ecuaciones de Navier-Stokes no se conoce después de realizada la simulación, debido a que aún no se ha logrado resolver tal sistema de ecuaciones, de manera que no existe un resultado analítico contra el cual comparar. De esta forma, la única manera de conocer la validez de los resultados que se obtienen en una simulación es por medio de la validación experimental. Teniendo esto presente, se ha planteado la construcción de un banco de pruebas para el turbocargador HOLSET HT3B que se encuentra en la Universidad Nacional de Colombia, lo cual se constituye como un proyecto fundamental para la caracterización del modelo elaborado en FLUENT $^{\circledR}$ y la verificación de la validez de los resultados obtenidos en el presente trabajo. Una vez que la validación de los resultados obtenidos por medio de la simulación arroje un resultado positivo, lo cual puede darse después de modificar los parámetros reportados en este trabajo, y solamente para un rango de flujo másico estrecho, como se ha visto en artículos como [4], será posible comenzar a usar el CFD como herramienta de diseño para compresores en los que las condiciones de funcionamiento varíen ligeramente con respecto a las de la turbomáquina objeto de la validación.

\section{AGRADECIMENTOS}

La simulación objeto de este artículo contó con el valioso apoyo de Marian Stella Pastrana Rojas, Arnold Escobar y Oscar Grandas de la Universidad de San Buenaventura - Bogotá, quienes con su experiencia en el manejo de FLUENT $^{\circledR}$ y la documentación que habían reunido con anterioridad en torno al tema, dieron un aporte valioso a la presente simulación. Gracias por su colaboración.

\section{REFERENCIAS}

[1] J.A. Acosta, C.A. Duque, J.M. Mantilla y C.H. Galeano. "Digitalización 3D del rodete de un compresor centrífugo: un procedimiento alternativo". Ingeniare. Revista chilena de ingeniería. Vol. 15 No 3, pp. 236-244. 2007.

[2] J.A. Acosta y C. A. Duque. "Planteamiento de una metodología para abordar la simulación de flujos en compresores centrífugos con CFD”. Proyecto de Grado. Departamento de Ingeniería Mecánica y Mecatrónica. Universidad Nacional de Colombia. Bogotá, Colombia. 2006.

[3] Fluent Inc. GAMBIT User's Manual. 2006.

[4] E. Dick, J. Vierendeels, S. Serbruyns and J.V. Voorde. "Perfomance prediction of centrifugal pumps with CFD-Tools". Seminar/Summer School CFD for Turbomachinery applications. Gdansk, Poland. Septiembre 01-03, 2001.

[5] M. Lobmaier. "Numerische Simulation der Strömung in einem Radialventilator mit hoher spezifischer Drehzahl". Institut für Thermodynamik und Energiewandlung, Technische Universität Wien. Viena, Austria. 2004.

[6] Fluent Inc. FLUENT User's Manual. 2006.

[7] J. B. Heywood. "Internal Combustion Engine Fundamentals". McGraw-Hill. 1988. 